\title{
AVALIAÇÃO DOS MODELOS DIGITAIS DE ELEVAÇÃO (MDE) DERIVADOS DE IMAGENS DE SENSORIAMENTO REMOTO ORBITAL
}

\section{EVALUATION OF DIGITAL ELEMENT MODELS (MDE) DERIVED FROM ORBITAL REMOTE SENSING IMAGES}

\author{
Renato Cardoso Teixeira ${ }^{1}$; Gustavo Henrique Mendes Brito ${ }^{2}$; Arlindo Modesto Antunes ${ }^{3}$; Ivandro José \\ De Freitas Rocha ${ }^{4}$

\begin{abstract}
${ }^{1}$ Engenheiro Agrônomo, renato.r.ct@hotmail.com
2Engenheiro Agrícola - Faculdade Evangélica de Goianésia, gh.mendes.brito@gmail.com, ${ }^{3}$ Engenheiro Agrícola - Universidade Federal Rural da Amazônia, arlindo.modesto1@hotmail.com ${ }^{4}$ Ciência da Computação - Faculdade Evangélica de Goianésia, ivandro_rocha@yahoo.com.br
\end{abstract}

Resumo: Modelos digitais de elevação, MDE, auxiliam em desenvolver atividades agrícolas como escolhas das áreas de atuação, cultura a ser introduzida, obter informações topográficas em locais de difícil acesso, traçar sentido de plantio, permite uma melhor distribuição e abertura de carreadores de acesso nas lavouras, fornece uma prévia do traçado de curvas em nível, também orienta em traçar um perfil longitudinal do solo de uma localização a outra. O objetivo deste trabalho foi analisar qual imagem de satélite possui informações que mais aproxima da real situação topográfica da área em estudo. Utilizando pontos GPS coletados em campo, juntamente com imagens de satélite ASTER, SRTM, TOPODATA e suas respectivas informações disponíveis. Foi extraído o erro médio quadrático e determinado qual imagem de satélite possui melhor resultado correlacionado com as elevações do local estudado. A imagem que gerou o menor valor RMSE foi o MDE TOPODATA, com valor de 12,59 m, passando a ser a informação que melhor refletiu o plano topográfico local. Á associação da atividade agrícola junto ao solo, se aplica ou adequa conforme as elevações do terreno.

Palavras-chave: Topografia, Declividade, GPS, Agricultura.

\begin{abstract}
Digital elevation models, MDE, help to develop agricultural activities as choices of areas of operation, culture to be introduced, topographic information in difficult to reach places, to draw a sense of planting, allows a better distribution and opening of access roads in the fields, provides a preview of level contouring, also guides in drawing a longitudinal profile of the ground from one location to another. The objective of this work was to analyze which satellite image has information that most closely approximates the actual topographic situation of the study area. Using GPS points collected in the field, together with ASTER, SRTM, TOPODATA satellite images and their respective available information, the quadratic root of the mean square error was extracted through the RMSE equation, determining which satellite image has the best result correlated with the elevations of the study site. The image that generated the lowest RMSE value was the TOPODATA MDE, with a value of $12.59 \mathrm{~m}$, becoming the information that best reflected the local topographic plan. The association of agricultural activity with the soil, is applied or adapted according to the terrain elevations.
\end{abstract}

Key words: Topography, Slope, GPS, Agriculture.

\section{INTRODUÇÃO}

A modelagem de um ambiente natural ou antrópico está relacionado com a topografia, especificamente os levantamentos planialtimétricos que descrevem o terreno com exatidão e levando em consideração as medidas planas, ângulos e inclinação do local a ser estudado (SCHIETTI et al., 2007). Atividades agrícolas, análises ambientais, cultivo em nível, sistematização de terrenos, desmembramento, remembramento, cálculo de área, entre outras aplicações, são importantes variáveis topográficas oriundas dos levantamentos planialtimétricos (VALERIANO e CARVALHO JUNIOR, 2003).

Embora as variáveis sejam importantes informações para auxiliar na tomada de decisão e 
planejamento do uso do solo, existe um déficit de mapeamentos planialtimétricos em grandes e médias escalas no Brasil, devido à complexidade na obtenção e processamento das informações topográficas nestas escalas (VALERIANO e ROSSETTI, 2011; SCHIETTI et al., 2007).

Uma alternativa para a obtenção de informações planialtimétricas em áreas de grandes escalas, é a utilização dos modelos digitais de elevação, MDE. Estes modelos consistem na representação altimétrica digital contínua de toda a área, distribuída espacialmente em variações de altitude, numa área baseada e definida sobre um plano cartográfico com coordenadas X, Y e Z. As coordenadas $Z$ representam valores da altitude e profundidade num modelo tridimensional (HENGL e MACMILLAN, 2009).

Entre estes modelos podemos destacar o Shutle Radar Topographic Mission (SRTM), Advanced Spaceborne Thermal Emission and Reflection Radiometer - Global Digital Elevation Model (ASTER) e TOPODATA, que disponibilizam informações de relevo por imagens de sensores remotos e técnicas de interpolação (SANTOS et al., 2006).

O SRTM possui uma acurácia vertical de cerca de 16 metros para a banda $C$ e de 6 metros para a banda $\mathrm{X}$, a acurácia planimétrica absoluta é da ordem de 20 metros na banda C e 15 metros para a banda $X$, utilizando um nível de confiança de $90 \%$, de acordo com a National Map Accuracy Standard (FARR et al., 2007).

ASTER (Advanced Spaceborne Thermal Emission and Reflection Radiometer - Global Digital Elevation Model), foi concebido através de uma parceria entre o Ministério de Economia, Industria e Comércio do Japão (METI) e a Agência Espacial Norte Americana (NASA). (FARR et al., 2007).

Através de imagens estereoscópicas com precisão planimétrica de 6 metros e absoluta de 12 metros, obtidas do sensor ASTER, presente no satélite "Terra" entre os anos de 2000 e 2010, foi gerado imagens espaciais de 1 segundo de arco ( $\sim 30 \mathrm{~m}$ no equador), no sistema geodésico WGS84 com associação ao modelo geoidal EGM96. (TACHIKAWA et al., 2011).

Derivado do modelo SRTM, o sistema TOPODATA, refinou a resolução espacial de 3 arco de segundos para 1 arco de segundo, interpolando pelo o método Krigagem e através de derivações geomorfológicas. A intenção foi melhorar a percepção de feições relativamente detalhadas (VALERIANO, 2008).

Neste sentido, o objetivo com este trabalho foi avaliar dentro de uma área agrícola no município de Goianésia, GO, a qualidade e precisão altimétrica de modelos digitais de elevação (MDE) obtidos de sensoriamento remoto orbital como subsídio a obtenção de variáveis topográficas em atividades agrícolas.

\section{MATERIAL E MÉTODOS}

\section{Caracterização da área de estudo}

A área de estudo possui está localizada na coordenada Longitude $-49^{\circ} 06^{\prime} 34,47^{\prime \prime}$ e Latitude $15^{\circ} 16^{\prime} 55,44^{\prime \prime}$ a $657 \mathrm{~m}$ acima do nível médio dos mares, situada às margens da Rodovia Estadual GO 438, trecho que liga os municípios de Goianésia, GO, ao município de Santa Rita do Novo Destino, GO, (FIGURA 1). 


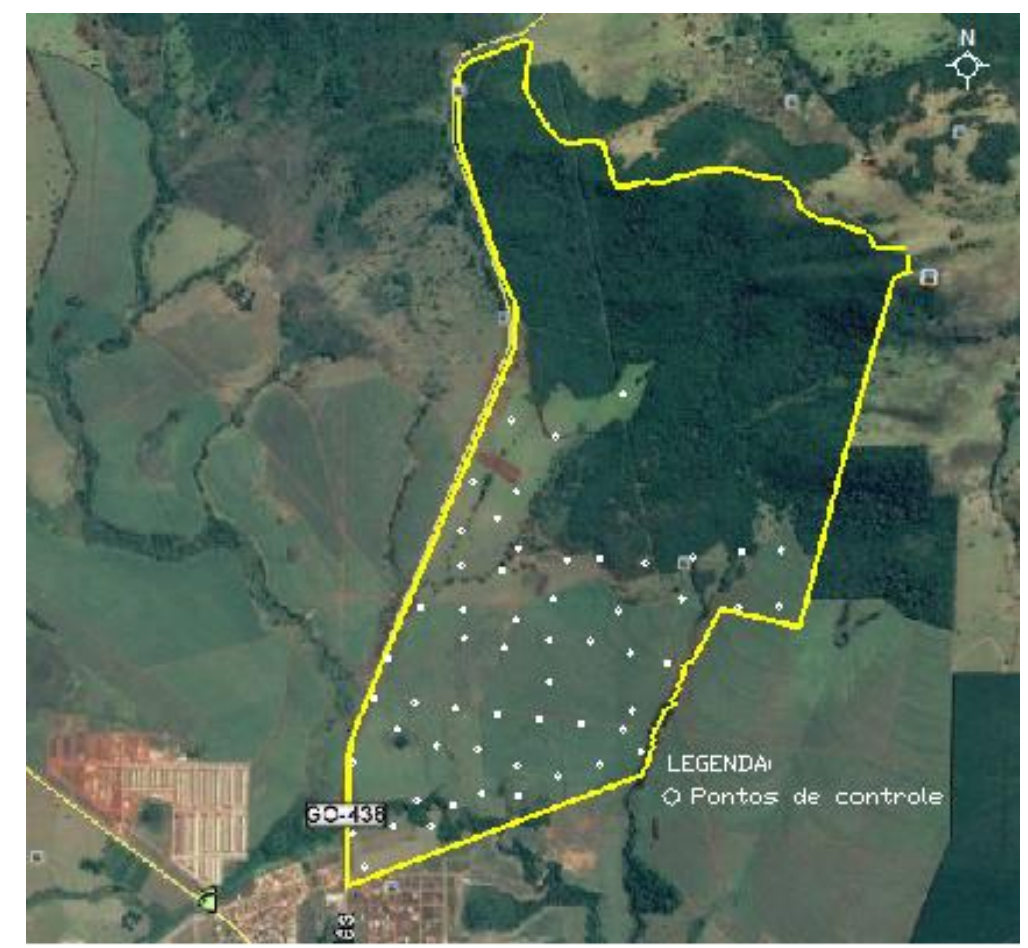

Figura 1. Localização da área avaliada e pontos de controle das altitudes obtidas por GPS de navegação no município de Goianésia, GO.

A vegetação predominante remanescente é do tipo cerradão, sendo árvores predominantes de porte alto e diâmetro do caule superior a $30 \mathrm{~cm}$. Existe formação de novos cerrados nas depressões periféricas. O restante do perímetro está composto por pastagens em capim colonião e atividades agrícolas.

\section{Aquisição dos modelos digitais de elevação}

Foram utilizados os modelos digitais de elevação ASTER, SRTM e TOPODATA, ambas com $30 \mathrm{~m}$ de resolução espacial, sendo ASTER e SRTM disponibilizados de maneira gratuita pelo United States Geological Survey - USGS, e o MDE do acervo TOPODATA, disponibilizado de maneira gratuita pelo Instituto Nacional de Pesquisas Espaciais - INPE (USGS, 2017; INPE, 2017).

Os dados cartográficos foram obtidos no sistema de coordenadas geográficas Datum WGS 84 e convertidos para sistema de coordenadas plano retangular

Mercator (UTM), fuso 22L.

\section{Análise da precisão altimétrica}

Utilizando GPS modelo Etrex 10, marca Garmim, foram levantados 56 pontos de controle configurados no sistema de coordenadas plano retangular Universal Transversa de Mercator (UTM), Datum WGS 84, fuso 22L, sempre na altura de 1,05 m em relação ao nível do solo. Os pontos de controle foram descarregados no Software TrackMaker obtendo as coordenadas e a altitude em relação ao nível médio dos mares, possibilitando verificar a precisão submétrica.

Para avaliar a qualidade dos Modelos Digitais de Elevação, foi utilizado a metodologia proposta por Chagas et al. (2010), através de uma equação. Essa equação traz a raiz quadrada do erro médio quadrático da elevação, RMSE. 


$$
R M S E=\sqrt{\frac{\sum_{i=1}^{n} d_{i}^{2}}{\mathrm{n}}}
$$

Onde "d" é a diferença de elevação entre os MDEs avaliados e os pontos de controle ao quadrado e " $n$ " é o número de pontos de elevação testados.

Para um bom aproveitamento da área e do solo, em práticas agrícolas, a utilização do RMSE ao avaliar os Modelos Digitais de Elevação torna possível verificar qual MDE representa melhor as elevações da área em estudo, devido compor nos dados, informações levantadas "in loco". O MDE que gerou o resultado RMSE menor, passou a ser o modelo mais adequado para as práticas agrícolas preliminares.

Os dados do MDE e os pontos de controle foram submetidos a um processo de correção, onde houve remoção das depressões espúrias e analise da declividade, com auxílio da função slope, que disponibiliza informações topográficas, disponível na extensão Spatial Analyst do software ArcGIS 10.1.

\section{RESULTADOS E DISCUSSÃO}

Comparando os MDEs ASTER, SRTM e TOPODATA (Tabela 1), os modelos TOPODATA e SRTM, possuem os menores valores RMSE sendo os modelos mais indicados para suas devidas aplicações. O modelo ASTER quando comparado aos demais MDEs, teve seu RMSE alto e uma aplicabilidade agrícola inadequada, que pode gerar informações de altitudes ou um relevo que não representa a realidade do plano topográfico local.

Tabela 1. Dados de elevação máxima, média e mínima, desvios e RMSE obtidos por levantamento in loco (GPS), MDE ASTER, SRTM e TOPODATA em Goianésia, GO.

\begin{tabular}{cccccc}
\hline & & & & DESVIO & \\
MDE & MAX $(\mathbf{m})$ & MIN $(\mathbf{m})$ & MED (m) & (m) & RMSE (m) \\
\hline ASTER & 1066 & 612 & 749,1798 & 107,1137 & 39,27 \\
SRTM & 1068 & 617 & 750,3448 & 107,1639 & 13,04 \\
TOPODATA & 1065,35 & 619,94 & 753,9878 & 109,5157 & 12,59 \\
\hline
\end{tabular}

Obtidos pela diferença das elevações dos MDEs e dos pontos GPS através do RMSE.

Resultados semelhantes foram encontrados por Silveira et al (2013), que compararam diferentes MDEs no estado do Paraná, constatando o RMSE variando entre 0,9 até 12,64 metros e imagens SRTM de 1 arco de segundo com menor erro quadrático médio.

Nos resultados obtidos do trabalho de Hengl e Macmillan (2009), as variações do RMSE ocorreram devido a resolução espacial das imagens, que foram variações de $30 \mathrm{~m}$ entre TOPODATA, ASTER e SRTM, ambas com 30 e $90 \mathrm{~m}$ de resolução espacial. A resolução espacial influencia consideravelmente no erro médio quadrático, por interferir na qualidade de visualização do radar ao exercer suas funções que é coletar dados da área desejada. (HIRANO et al., 2003; CUARTERO et al., 2004; POLI et al., 2004). 
Entretanto o presente trabalho avaliou apenas MDEs com $30 \mathrm{~m}$ de resolução espacial, deste modo, as respostas do RMSE, pode estar associado as características do terreno, como áreas de encostas, presença voçorocas, área com o ponto mais baixo e o mais alto possuindo uma variação grande em curta distância, caracterizando terreno íngreme.

No momento em que o satélite passou pela área em estudo pode ter ocorrido um posicionamento inadequado ou presença de nuvens, sendo outro fator que influencia no resultado do RMSE.

Hodgson et al. (2003) citam comparações de MDEs em uma área com porcentagem de inclinação e 0 a 14\%, onde foi analisado erros de elevações em área íngremes, com plantas de porte alto e baixo. Os resultados demostraram que áreas em grandes elevações, solo com determinado tipo de cobertura, como grama baixa e alta, pinheiro e áreas bem arbustivas, mascara as informações.

A forma de obtenção das imagens também pode ter interferido no RMSE elevado da imagem ASTER, pois a mesma é obtida de forma estereoscópica e as imagens SRTM e TOPODATA por Interferometria. Estereoscopia defini por fotografar a face dos relevos e Interferometria, por utilizar ondas sobrepostas que criam novas ondas representado os relevos nas áreas desejadas, o que torna o método da Interferometria ser o mais preciso em relação ao método de Estereoscopia (MOREIRA, 2011).

Utilizando as elevações dos modelos ASTER, SRTM e TOPODATA, foi observado a declividade média da área em estudo, sendo 16,5\%, 15,25\% e 14,75 respectivamente (FIGURA 2). O modelo ASTER apresentou uma declividade maior em relação aos demais modelos e isso pode ter interferido na qualidade do RMSE.

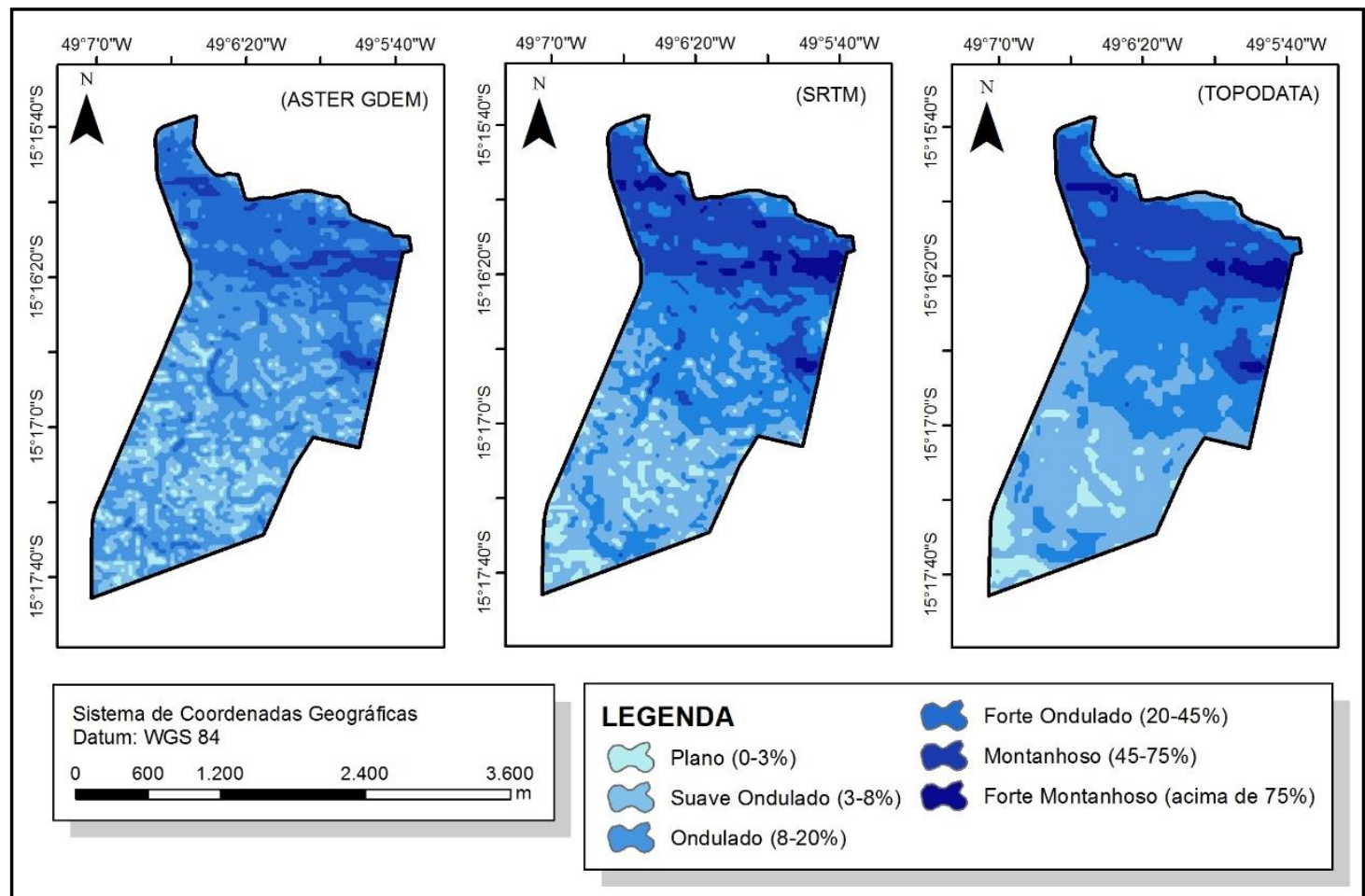

Figura 2. Mapa de declividade derivados dos modelos digitais de elevação. 
Estudos mostram que as características do terreno, sendo inclinações, elevações em relação ao nível médio dos mares, altitudes variadas e o método de obtenção das imagens de satélites, interferem nas informações RMSE.

Segundo Kocak et al. (2004) e Gerstenecker et al. (2005), a inclinação do terreno interfere na exatidão dos MDEs derivados de sensores remotos, onde há uma grande dependência em relação a inclinação do terreno.

Em áreas com altitudes elevadas $\mathrm{e}$ extremamente inclinadas em relação ao plano horizontal, Falorni et al. (2005) recomendam o valor RMSE sempre menor ou igual a $16 \mathrm{~m}$ para MDEs e que os MDEs sempre devem ser considerados apenas como uma referência.

\section{CONCLUSÕES}

O MDE TOPODATA e SRTM são recomendados para derivar atributos topográficos primários ou secundários, empregadas no estudo das elevações da área avaliada no município de Goianésia, GO.

O RMSE pode ser utilizado satisfatoriamente como critério para seleção de MDEs, com base em altitudes rastreadas in loco, com uso de aparelho GPS topográfico.

O MDE ASTER apresenta menor precisão em representar o plano topográfico local da área avaliada.

\section{REFERÊNCIAS BIBLIOGRÁFICAS}

CHAGAS, C. S.; FERNANDES FILHO, E. I.; ROCHA, M. F.; CARVALHO JUNIOR, W.; SOUZA NETO, N. C. Avaliação de modelos digitais de elevação para aplicação em um mapeamento de digital de solos. Revista
Brasileira de Engenharia Agrícola e Ambiental, v.14, n.2, p.218-226, 2010.

CUARTERO, A.; FELICÍSIMO, A. M.; ARIZA, F. J. Accuracy of DEM generation from TerraAster stereo data. International Archives of Photogrammetry and Remote Sensing, v.35, p.225-260, 2004.

FARR, T. G.; ROSEN, P. A.; CARO, E.; CRIPPEN, R.; DUREN, R.; HENSLEY, S.; KOBRICK, M.; PALLER, M.; RODRIGUEZ, E.; ROTH, L.; SEAL, D.; SHAFFER, S.; SHIMADA, J.; UMLAND, J.; WERNER, M.; OSKIN, M.; BURBANK, D.; ALSDORF, D. The Shuttle Radar Topography Mission. Reviews of Geophysics, v.45, p.1-33, 2007.

FALORNI, G.; TELES, V.; VIVONI, E. R.; BRAS, R. L.; AMARATUNGA, K.S. Analysis and characterization of the vertical accuracy of digital elevation models from the Shuttle Radar Topography Mission. Journal of Geophysical Research, v.110, p.1-20, 2005.

GERSTENECKER， C.; LÄUFER， G.; STEINECK, D.; TIEDE, C.; WROBEL, B. Validation of digital elevation models around Merapi Volcano, Java, Indonesia. Natural Hazards and Earth System Sciences, v.5, p.863-876, 2005.

HENGL, T.; MACMILLAN, R. A. Geomorphometry - A Key to Landscape Mapping and Modelling. In: HENGL, T.; REUTER, H. I. (eds.) Geomorphometry Concepts, Software, Applications, Series Developments in Soil Science vol. 33, Amsterdam: Elsevier, pp. 433-460, 2009.

HIRANO, A.; WELCH, R.; LANG, H. Mapping from ASTER stereo image data: DEM validation and accuracy assessment. ISPRS Journal of Photogrammetry e Remote Sensing, v.57, p.356-370, 2003.

HODGSON, M. E.; JENSEN, J. R.; SCHMIDT, L.; SCHILL, S.; DAVIS, B. An evaluation of LIDAR- and IFSAR-derived digital elevation models in leaf-on conditions with USGS level 1 and level 2 DEMs. Remote Sensing of Environment, v. 84, p. 295-308, 2003. 
KOCAK, G.; BÜYÜKSALIH, G.; JACOBSEN, K. Analysis of digital elevation models determined by high resolution space images. International Archives of Photogrammetry and Remote Sensing, v.35, p.636-641, 2004.

MOREIRA, M. A., Fundamentos do sensoriamento remoto e metodologias de aplicação - 4. ed. atual.e ampl.- Viçosa, MG: Ed. UFV, 2011.

POLI, D.; REMONDINO, F.; DOLCI, C. Use of satellite imagery for DEM extraction, landscape modeling and GIS applications. In: International Workshop on Processing and Visualization Using High-Resolution Imagery, 2004, Pitsanulok. Proceedings... Pitsanulok: ISPRS, 2004

SANTOS, L.J.C.; OKA-FIORI, C.; CANALI, N.E.; FIORI, A.P.; SILVEIRA, C.T.; SILVA, J.M.F.; ROSS, J.L.S. Mapeamento geomorfológico do Estado do Paraná. Revista Brasileira de Geomorfologia. n2, p. 3-12, 2006.

SILVEIRA, C. T.; FIORI, A. P.; FERREIRA, A. M.; GÓIS, J.R.; MIO, G.; SILVEIRA, R. M. P.; MASSULINI, N. E. B.; LEONARDI, T. M. H. Emprego de atributos topográficos no mapeamento da susceptibilidade a processos geoambientais na bacia do rio Jacareí/Paraná. Sociedade e Natureza, v.24, p. 623-639, 2013.
SCHIETTI, J.; DRUCKER, D.; KEIZER, E.; CARNEIRO FILHO, A.; MAGNUSSON, W. 2007. Avaliação do uso de dados SRTM para estudos ecológicos na Amazônia central. Simpósio Brasileiro de Sensoriamento Remoto, 13, Florianópolis, Brasil, Anais..., p. 6949-6955, 2007.

TACHIKAWA, T.; KAKU, M.; IWASAKI, A.; G ESCH, D.; OIMOEN, M.; ZHANG, Z.; DAN IELSON J., KRIEGER, T.; CURTIS, B.; HA ASE, J.; ABRAMS, M.; CRIPPEN, R.; CARA BAJAL, C. 2011. ASTER Global Digital Elevation Model Version 2-Summary of Validation Results, 2011.

VALERIANO, M. M., ROSSETTI, D. F. Topographic modelling of Marajó island with SRTM data. Revista Brasileira de Geomorfologia, v.9, p.53-64, 2008.

VALERIANO, M. M.; ROSSETTI, D.F. TOPODATA: Brazilian full coverage refinement of SRTM data. Applied Geography, v.32, n.2, p.300-309, 2011.

VALERIANO, M. M.; CARVALHO JÚNIOR, O, A. Geoprocessamento de Modelos digitais de Elevação para Mapeamento da Curvatura Horizontal em Microbacias. Revista Brasileira de Geomorfologia, v.4, n.1, p.17-29. 2003. 